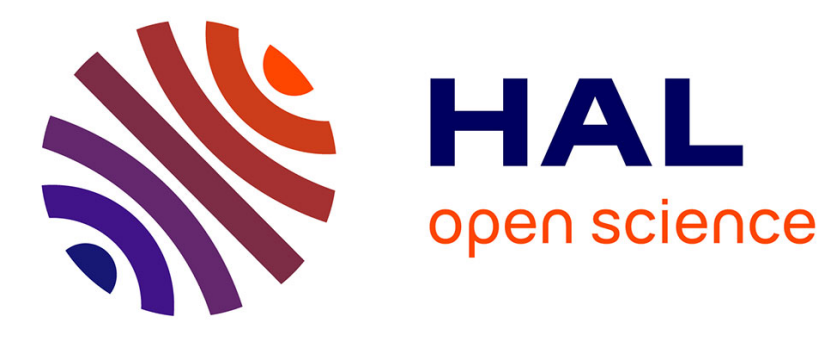

\title{
Stability of three-phase ternary-eutectic growth patterns in thin sample
}

S Bottin-Rousseau, M S erefog lu, Sinan Yücetürk, Gabriel Faivre, Silvère

Akamatsu

\section{- To cite this version:}

S Bottin-Rousseau, M S erefog ${ }^{`}$ lu, Sinan Yücetürk, Gabriel Faivre, Silvère Akamatsu. Stability of three-phase ternary-eutectic growth patterns in thin sample. Acta Materialia, 2016, 109, pp.259 - 266. 10.1016/j.actamat.2016.01.065 . hal-01472485

\section{HAL Id: hal-01472485 \\ https://hal.science/hal-01472485}

Submitted on 20 Feb 2017

HAL is a multi-disciplinary open access archive for the deposit and dissemination of scientific research documents, whether they are published or not. The documents may come from teaching and research institutions in France or abroad, or from public or private research centers.
L'archive ouverte pluridisciplinaire HAL, est destinée au dépôt et à la diffusion de documents scientifiques de niveau recherche, publiés ou non, émanant des établissements d'enseignement et de recherche français ou étrangers, des laboratoires publics ou privés. 


\title{
Stability of three-phase ternary-eutectic growth patterns in thin sample
}

\author{
Sabine Bottin-Rousseau ${ }^{\mathrm{a}}$, Melis Şerefoğlu ${ }^{\mathrm{b}, *}$, Sinan Yücetürk $^{\mathrm{b}}$, Gabriel Faivre $^{\mathrm{a}}$, \\ Silvère Akamatsu ${ }^{\mathrm{a}}$ \\ ${ }^{a}$ Sorbonne Universités, UPMC Univ Paris 6, CNRS-UMR 7588, Institut des NanoSciences de \\ Paris, case courrier 840, 4 place Jussieu, 75252 Paris Cedex 05, France \\ ${ }^{b}$ Department of Mechanical Engineering, Koç University, Rumeli Feneri Yolu, 34450 Sariyer, \\ Istanbul, Turkey
}

\begin{abstract}
Near-eutectic ternary alloys subjected to thin-sample directional solidification can exhibit stationary periodic growth patterns with an ABAC repeat unit, where $\mathrm{A}, \mathrm{B}$ and $\mathrm{C}$ are the three solid phases in equilibrium with the liquid at the eutectic point. We present an in-situ experimental study of the dynamical features of such patterns in a near-eutectic $\mathrm{In}-\mathrm{In}_{2} \mathrm{Bi}-\mathrm{Sn}$ alloy. We demonstrate that ABAC patterns have a wide stability range of spacing $\lambda$ at given growth rate. We study quantitatively the $\lambda$-diffusion process that is responsible for the spacing uniformity of steady-state patterns inside the stability interval. The instability processes that determine the limits of this interval are examined. Qualitatively, we show that ternary-eutectic ABAC patterns essentially have the same dynamical features as two-phase binary-eutectic patterns. However, lamella elimination (low- $\lambda$ stability limit) occurs before any Eckhaus instability manifests itself. We also report observations of stationary patterns with an $[\mathrm{AB}]_{\mathrm{m}}[\mathrm{AC}]_{\mathrm{n}}$ superstructure, where $m$ and $n$ are integers larger than unity.
\end{abstract}

Keywords: Eutectic solidification, directional solidification, solidification microstructures, ternary eutectics, three phase microstructures.

\footnotetext{
*Corresponding author

Email address: mserefoglu@ku.edu.tr (Melis Şerefoğlu )
} 


\section{Introduction}

The solidification microstructures of ternary eutectic alloys take many different forms depending on the composition and grain structure of the alloy, the geometrical and thermal features of the solidification device, and the solidification history. The most important of these factors are the number of growing phases and the dimensionality of the samples. We are concerned here with two-dimensional three-phase microstructures. These are typically observed in near-eutectic ternary alloys subjected to thin-sample directional solidification (thin-DS). Solidification microstructures are, we recall, nothing else than the trace left behind in the solid by out-of-equilibrium self-organized patterns formed during solidification. At constant solidification rate $V$ and applied thermal gradient $G$, these patterns generally reach, or, at least, asymptotically tend towards a steady-state. The most wellknown example of a steady-state eutectic growth pattern is the periodic (lamellar) two-phase solidification pattern of near-eutectic binary alloys analyzed by Jackson and Hunt $(\mathrm{JH})$ a long time ago [1]. The repeat unit of such a pattern is an $\mathrm{AB}$ pair of lamellae, where A and $\mathrm{B}$ are the two solid phases in equilibrium with the liquid at the eutectic point. These $\mathrm{AB}$ patterns have mirror symmetry with respect to the mid-plane of the lamellae, which is actually a condition for their steadiness. Regarding ternary eutectic alloys, the basic repeat unit of stationary thin-DS patterns is $\mathrm{ABAC}$, where $\mathrm{A}, \mathrm{B}$ and $\mathrm{C}$ are the three eutectic solid phases. It has mirror symmetry with respect to the mid-plane of the $\mathrm{B}$ and $\mathrm{C}$ lamellae (Fig. 1). This was previously highlighted by Witusiewicz and coworkers in the $\mathrm{In}_{-}-\mathrm{In}_{2} \mathrm{Bi}-\mathrm{Sn}$ alloy [2] and a transparent organic alloy [3], and numerically demonstrated by Choudhury et al. [4] (also see Refs. [5, 6] regarding bulk solidification). However, the large-scale dynamics of the ABAC patterns has not yet been studied.

Here we present an experimental study of the morphological stability of ABAC

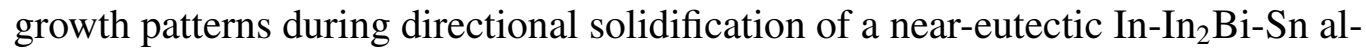
loy. We used very thin $(\approx 13 \mu \mathrm{m}$ thick) samples in order for the system to be quasi two-dimensional and rid of convection flows in the liquid. Quantitative results that are presented below were obtained by studying in real time the dynamic response of pre-uniformized ABAC patterns to upward or downward $V$-jumps. Qualitatively, the results may be best understood through a comparison with the known dynamical features of the binary $\mathrm{AB}$ patterns $[1,7,8,9,10]$ : (i) binary $\mathrm{AB}$ patterns have a wide stability range of spacing $\lambda$ at given $V$; (ii) any spatial variation of $\lambda$ that is confined within the stability interval is damped out over time through a long-range process called spacing-diffusion or $\lambda$-diffusion, which leads asymptotically to a perfectly periodic (uniform) pattern; (iii) the upper limit 
of the $\lambda$-range of stability $\left(\lambda_{\text {sup }}\right)$ is due to the onset of oscillations leading, at still larger $\lambda$-values, to lamella splitting; (iv) the lower stability limit $\left(\lambda_{\text {inf }}\right)$ corresponds to an instability of the $\lambda$-diffusion process, namely, a change of sign of the $\lambda$-diffusion coefficient (sometimes referred to as an Eckhaus instability [11]), which leads to lamella elimination; (v) $\lambda_{\text {sup }}$ varies with $V$ as $V^{-1 / 2}$ and is independent of $G$; in other words, it obeys the well-known Jackson and Hunt (JH) scaling law $\lambda_{\text {sup }} \propto \lambda_{m}$, where $\lambda_{m}$ is a scaling length that varies with $V$ as $V^{-1 / 2}$; (vi) $\lambda_{\text {inf }}$ does not obey the JH scaling law, but depends on both $V$ and $G$ in such a way that, at fixed $G$, the width of the stability range relative to $\lambda_{m}$, i.e. $\left(\lambda_{\text {sup }}-\lambda_{\text {inf }}\right) / \lambda_{m}$, increases as $V$ decreases. In the following, we shall call spacing, and denote it by $\lambda$, the width of an ABAC repeat unit in a three-phase eutectic-growth pattern. Which of the features listed above hold true for ternary-eutectic ABAC patterns? The main results of this study may be summed up as follows: ternary ABAC patterns have the same dynamical features as binary AB patterns, except for one significant aspect, namely, lamella elimination is not provoked by an Eckhaus instability but by a qualitatively different phenomenon, most probably, some short-wavelength instability of the ABAC pattern.

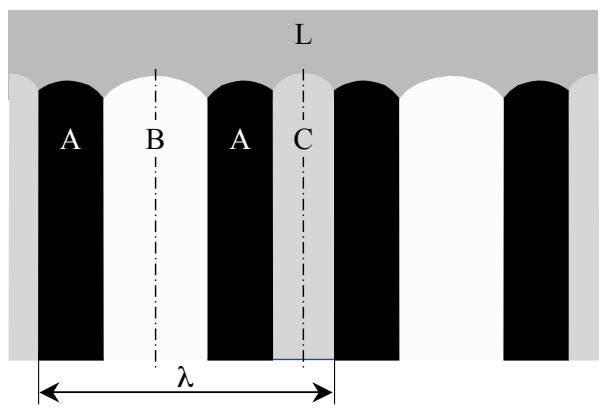

Figure 1: Sketch of the three-phase ABAC repeat unit of a ternary-eutectic directionalsolidification pattern. The thermal gradient is oriented vertically. In a thin sample of the eutectic In- $\mathrm{In}_{2} \mathrm{Bi}$-Sn alloy, the letters $\mathrm{A}, \mathrm{B}$, and $\mathrm{C}$ refer to the $\mathrm{In}_{2} \mathrm{Bi}, \beta$-In, and $\gamma$-Sn crystal phases, respectively. L: liquid. $\lambda$ : local spacing value.

In the remainder of the text, we first present the experimental methods (Section 2). We then give some important details about the preparation of extended ABAC lamellar patterns in large eutectic grains (Section 3). The main results (stability diagram and instability mechanisms, complex patterns) are presented and discussed in Section 4. A conclusion is proposed in the last section. 


\section{Experimental methods}

The In-Bi-Sn ternary phase diagram has a nonvariant eutectic point at the temperature of $332 \mathrm{~K}\left(T_{E}\right)$ and the composition of In-20.7at\%Bi-19.1at\% Sn $\left(C_{E}\right)$ [12]. The solid phases in equilibrium with the liquid at this point are the intermetallic compound $\operatorname{In}_{2} \mathrm{Bi}$, the $\beta$-In phase and the $\gamma$-Sn phase. For brevity, we will generally call these phases $\mathrm{A}, \mathrm{B}$, and $\mathrm{C}$, respectively. $\mathrm{In}_{2} \mathrm{Bi}$ has a hexagonal structure; $\beta$-In and $\gamma$-Sn have body-centered tetragonal structures. An alloy of nominal composition $C_{E}$ was prepared by weighting the appropriate quantities of $99.999 \%$ pure indium, bismuth and tin (Goodfellow), and mixing them in the liquid state under a primary vacuum. The actual composition was within less than 0.05 at $\%$ of $C_{E}$. Glass-wall samples with inner dimensions of $4 \times 50 \times 0.013 \mathrm{~mm}^{3}$ were filled with molten alloy using a vacuum-suction method.

Details about the thin-DS stage and the method of observation used can be found elsewhere $[13,10]$. Let us simply mention that the thin-DS stage is basically made of two temperature-regulated copper blocks separated by a 5-mm gap. The thermal gradient in the region of the solidification front is $G=8 \pm 0.9 \mathrm{Kmm}^{-1}$. The growth direction $\mathbf{z}$ is parallel to the thermal gradient and opposite to the pulling direction. The $\mathbf{y}$-axis is perpendicular to the sample plane, whereas the $\mathbf{x}$-axis is parallel to the isotherms. The $V$-range explored is $0.01-0.8 \mu m s^{-1}$. The solidification front is observed in real time in the $\mathbf{y}$ direction (side view) with a reflectedlight optical microscope (Leica DMI 5000) equipped with a monochrome digital camera (Scion), connected to a PC for image capturing, processing and analysis. With this method, what is actually observed is the surface of contact between the metallic film and a flat glass wall. After contrast enhancing, the $\beta$-In/glass surfaces appeared white and the $\mathrm{In}_{2} \mathrm{Bi} / \mathrm{glass}$ surfaces black; both the $\gamma$-Sn/glass and the liquid/glass surfaces appeared light-grey (Fig. 1). We also performed ex-situ metallographic observations of transverse cross-sections in some samples. These showed that the interphase boundaries were perpendicular to the glass walls, as expected, validating the use of side-view images for dynamical studies of isotropic grains, as we explain shortly.

\section{Extended ABAC lamellar patterns}

Concerning binary $\mathrm{AB}$ eutectic patterns, it is known that near-uniform eutectic patterns can only be grown in samples consisting of "floating" eutectic grains with a size much larger than the spatial average of $\lambda$. To explain the origin for this requirement, we must recall the following facts [14, 15]: (i) a (eutectic) grain 
is a portion of the solid, inside which the crystal-lattice orientation of each of the eutectic phases is uniform; (ii) eutectic growth patterns are sensitive to the degree of anisotropy of the surface energies of the various interfaces present, especially, $\mathrm{AB}$ interphase boundaries; this degree of anisotropy depends on the orientation of the different phases with respect to one another and the sample, and therefore varies from grain to grain; (iii) eutectic grains can be classified into two broad categories called "floating" and "locked". The floating grains are those in which anisotropy effects are sufficiently weak for the dynamical features of the eutectic patterns to be those that are reviewed in the Introduction (including, in particular, uniformisation over time by $\lambda$-diffusion). In locked grains, on the contrary, surface tension anisotropy has dramatic effects on the pattern formation. These grains most probably have a special orientation relationship between $\mathrm{A}$ and $\mathrm{B}$, and lowenergy planes for the $\mathrm{AB}$ boundaries. The interphase boundaries become locked onto these low-energy directions entailing that $\lambda$-diffusion is blocked; (iv) the boundaries between floating eutectic grains are a source of perturbations (lamella terminations, long-range spacing gradients) preventing the growth pattern to approach a fully steady state [14]. The same considerations apply to ternary ABAC patterns. In this case, however, three different orientation relationships $(A B, B C$, $\mathrm{CA}$ ) come into play. The floating grains are those, in which the $\mathrm{AB}, \mathrm{BC}$ and $\mathrm{CA}$ boundaries have weak anisotropy.

A large floating ABAC grain in near-eutectic $\mathrm{In}-\mathrm{In}_{2} \mathrm{Bi}-\mathrm{Sn}$ is shown in Fig. 2. The experimental procedure used to create such grains was as follows. After partial directional melting, the system was maintained at rest $(V=0)$ in order to let it return to equilibrium. During this period, two thin solid layers progressively formed between the liquid and the unmelted part of the solid: a polycrystal layer of $\mathrm{C}$ (i.e. $\gamma$-Sn) in equilibrium with the liquid, and, immediately below it, a twophase layer of $\mathrm{AC}$ (i.e. $\mathrm{In}_{2} \mathrm{Bi}+\gamma-\mathrm{Sn}$ ). This indicated that the actual composition of the alloy was slightly off-eutectic with a small excess of $\mathrm{Sn}$ and an even smaller excess of $\mathrm{Bi}$. The deviation of the alloy composition from $C_{E}$ was estimated from the observed phase fractions of $\mathrm{A}, \mathrm{B}$ and $\mathrm{C}$ during stationary growth to be less than 0.05 at $\%$. Immediately after the onset of the pulling, the solid-liquid interface recoiled along the $\mathbf{z}$-axis due to solute redistribution until a few AC grains overgrew the $C$ layer through an invasion/splitting process (see Ref. [16]). We repeated this melting-annealing-invasion procedure several times in order to decrease the number of AC grains. We then performed a two-phase (univariant) AC solidification until a three-phase solid appeared and overgrew the AC-liquid front, as previously reported by Rex et al. [17]. Figure 3a shows the tip region of a three-phase solid invading the surface of a floating AC eutectic grain. In the wake 
of the invasion, lamella splitting generated a disordered three-phase pattern with a very small spacing, which slowly reorganized through lamella elimination and $\lambda$-diffusion (Fig.3b) and eventually formed a regular ABAC pattern.

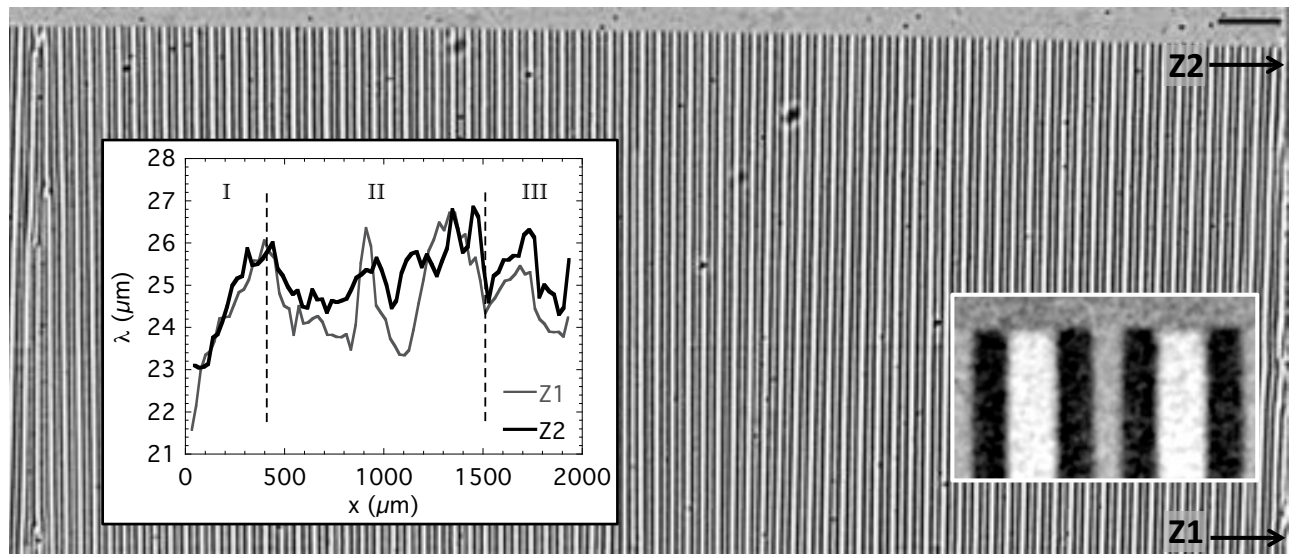

Figure 2: Near steady-state $\mathrm{In}_{-} \mathrm{In}_{2} \mathrm{Bi}-\mathrm{Sn}$ solidification pattern. $V=0.35 \mu \mathrm{ms}^{-1}$. Bar: $100 \mu \mathrm{m}$. Leftmost inset: $\lambda(x)$-plots at the $\mathrm{Z} 1$ and $\mathrm{Z} 2$ time points. The subdivision of the field of view into three areas along the $\mathbf{x}$ axis is explained in the text. Rightmost inset: close-up view of an ABAC pattern at $V=0.11 \mu \mathrm{ms}^{-1}$ (Horizontal dimension: $90 \mu \mathrm{m}$ ).

In the case of the experiment of Fig. 2, the sample contained two distinct floating grains, both of about $2 \mathrm{~mm}$ of lateral extension (one of them is shown in the figure). The boundary between the two grains is visible on the left-hand side, and the contact with the edge of the sample on the right-hand side of Fig. 2. The subdivision of the field of view into three areas indicated in the (left) inset is justified by the presence of two low-angle grain boundaries. All these defects generated lamella termination events and spacing gradients in their vicinity (regions I and III). However, and more importantly, the ABAC pattern inside the unperturbed region II clearly underwent a $\lambda$-diffusion smoothing process. The quantitative studies reported below were performed in this type of regions. We noticed a slight outward curvature of the isotherms (radius of curvature is about $10 \mathrm{~cm}$ ), but this had no detectable effect on the growth dynamics. We checked that the volume fractions of the eutectic phases measured in the micrographs did not vary with $x$ or $\lambda$, and were close to nominal values $\left(f_{B}=0.475, f_{C}=0.185\right)$. 

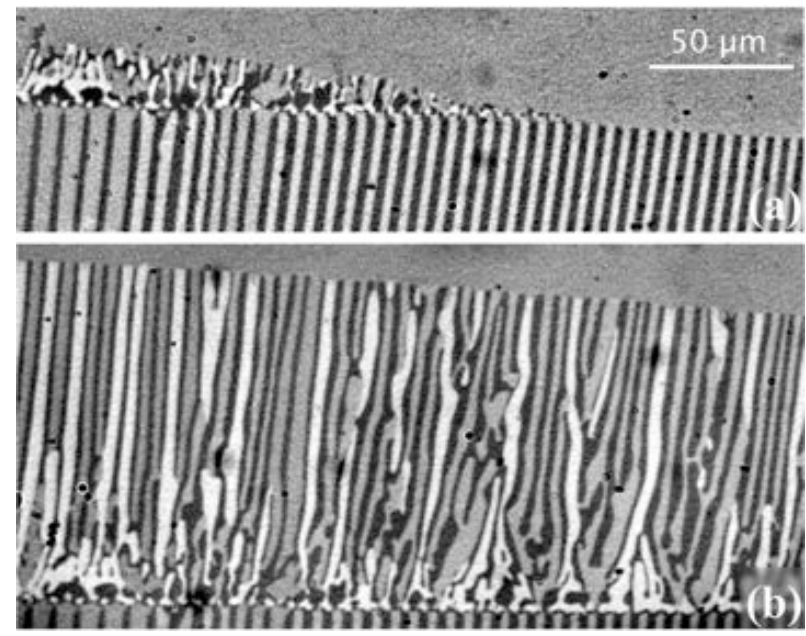

Figure 3: a) Invasion of an AC two-phase growth front by a three-phase solid during the early stages of thin-DS. $V=0.53 \mu \mathrm{ms}^{-1}$. b) Snapshot of the same area 300s later (see the early formation of an $\mathrm{ABAC}$ pattern on the left). The pronounced curvature of the growth front is due to the fact that it has not yet grown out of the funnel-shaped crystal selector placed at the cold end of the sample.

\section{Results and discussion}

\subsection{Stability diagram}

Each experimental run basically consisted of growing a near steady-state ABAC lamellar pattern at a given pulling velocity and then applying one, or a series of, upward or downward $V$-jumps. The initial pattern was characterized by its average spacing value $\lambda_{0}$ (equal to the width of the region of interest divided by the number of repeat units in this region). After a $V$-jump, the pattern either remained stable over a sufficiently long period of time or exhibited some instabilities. Downward $V$-jumps revealed only one kind of instability, namely, lamella termination, leading, self-evidently, to an increase in average spacing. Upward $V$-jumps gave rise to a more complex response combining oscillations and lamella splitting. We will first leave aside the oscillations, and assume that $\lambda_{\text {inf }}$ coincides with the threshold $\lambda_{e l}$ for the occurrence of lamella elimination, and $\lambda_{\text {sup }}$ with the threshold $\lambda_{b r}$ for lamella splitting. To construct a stability diagram, we represented any near steadystate $\mathrm{ABAC}$ pattern studied by a point in the $\lambda-V$ coordinates using different symbols according to the long-term evolution of the pattern (stable pattern, lamella splitting, lamella elimination). As shown in Fig. 4a, this revealed the existence of two clearly distinct stability limits, namely, $\lambda_{e l}(V)$ and $\lambda_{b r}(V)$. All the observed 
patterns that initially had a spacing distribution lying within these limits remained stable for the duration of the experiment. As an illustration, Fig. 5 shows two stable ABAC patterns at the same $V$-value, with $\lambda$-values that differ by a factor of about 1.4. Incidentally, we note that, in this example, the highest $\lambda$-value was only slightly smaller than $\lambda_{b r}$ and the pattern exhibited externally-sustained oscillations. We also plotted the data in terms of the dimensionless variable $\Lambda=\lambda / \lambda_{m}$, where $\lambda_{m}=K_{m} V^{-1 / 2}$ and $K_{m}=11.6 \mu \mathrm{m}^{3 / 2} \mathrm{~s}^{-1 / 2}$ (see below). As can be seen in Fig. $4 \mathrm{~b}, \Lambda_{b r}(\approx 1.9)$ was independent of $V$, within experimental uncertainty. In other words, $\lambda_{b r}$ obeyed the JH scaling law. By contrast, $\lambda_{e l}$ clearly deviated from this law at the lowest $V$-values explored. This is qualitatively similar to what was observed in binary $\mathrm{AB}$ patterns.

\subsection{Instability mechanisms}

Figure 6 shows a typical example, in which two successive upward $V$-jumps triggered oscillations and lamella splitting. The oscillations were coherent on a large scale and clearly belonged to an oscillatory mode which preserves the spatial period $(1 \lambda \mathrm{O})$. We did not observe any other mode of oscillation during this study. In Fig. 6, a $1 \lambda \mathrm{O}$ oscillation transiently appeared after a first upward $V$ jump and then was damped out. The second upward $V$-jump reinstalled the $1 \lambda \mathrm{O}$ oscillations, which amplified over time until a series of lamella splitting events occurred, thus decreasing $\lambda_{0}$ and killing the oscillations. It is worth noting that the attenuation/amplification times after the two $V$-jumps were of the same order of magnitude. This is consistent with the view that $\lambda_{\text {sup }}$ actually corresponded to the instability threshold spacing for the $1 \lambda O$ mode, as is the case in near-eutectic $\mathrm{AB}$ patterns in binary alloys $[7,8]$. However, contrary to what occurs in binary eutectics, there does not exist any stability domains of the $1 \lambda \mathrm{O}$ oscillations in the ABAC patterns. Concerning lamella splitting, little can be said of the underlying mechanism, which is intrinsically 3D. On the other hand, we note that each lamella splitting event resulted in the creation of a single new AB grain disrupting the ABAC stacking. In the example of Fig. 6, the motif of the resulting pattern is no longer $\mathrm{ABAC}$ but $\mathrm{ABABAC}$, or, more briefly, $[\mathrm{AB}]_{2} \mathrm{AC}$. We will discuss the stability of such complex patterns later on. Incidentally, it can be deduced from Fig. 6 that the error made by merging $\lambda_{\text {sup }}$ with $\lambda_{b r}$ was $10 \%$, or less.

The mechanism of lamella elimination showed some variability, as illustrated by the two examples of Fig. 7. Nevertheless, the following features were common to all the examples studied: (i) the process was strictly local before the elimination event occurred, but, on the contrary, gave rise to a long-range $\lambda$-diffusion 

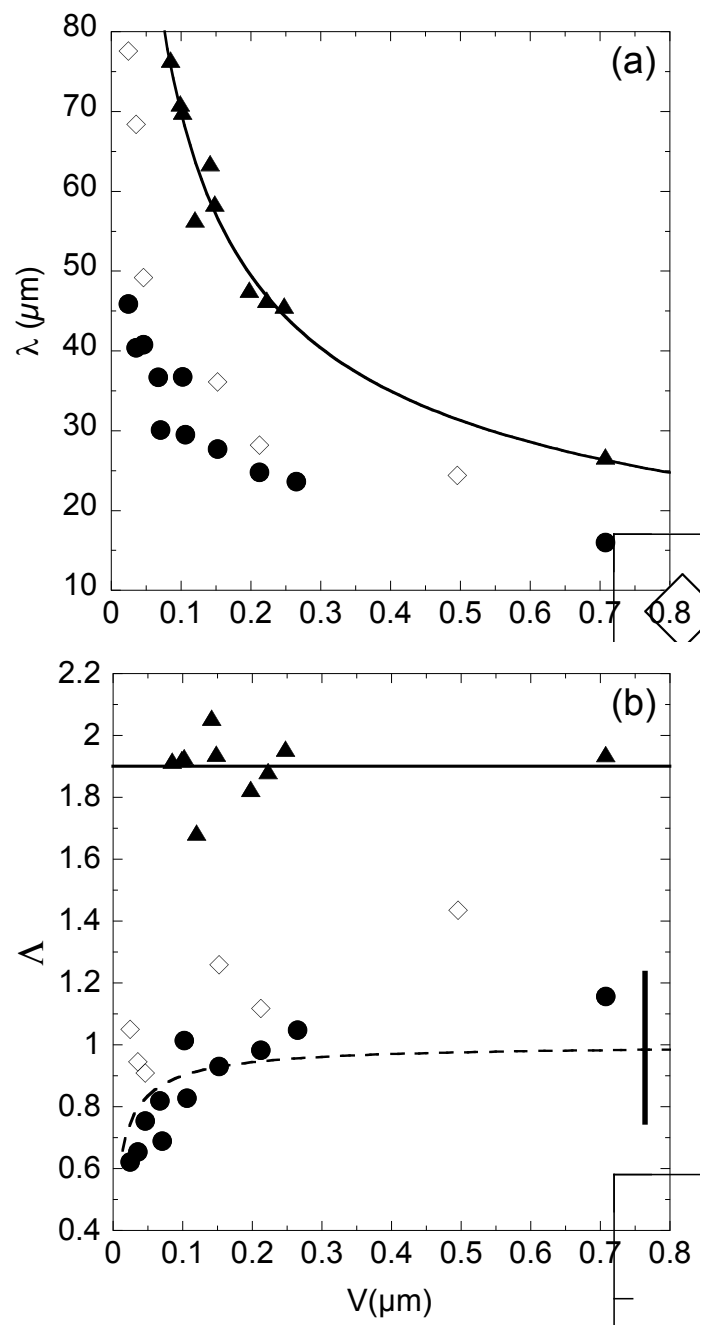

Figure 4: Stability diagram of the ABAC lamellar patterns: (a) spacing $\lambda$, and (b) dimensionless spacing $\Lambda=\lambda / \lambda_{m}$ of the pattern (see text), as a function of velocity $V$. Triangles: lamella splitting. Circles: lamella elimination. Diamonds: six stable patterns, in which the $\lambda$-diffusion coefficient was measured (see Table 1). Continuous line: best-fitting $\lambda_{b r} \propto V^{1 / 2}$ curve. Broken line: the $\lambda$-diffusion instability threshold $\lambda_{c}$ as given by Eq. 6 with $D_{\lambda}=0$. Vertical bar: error margin. 

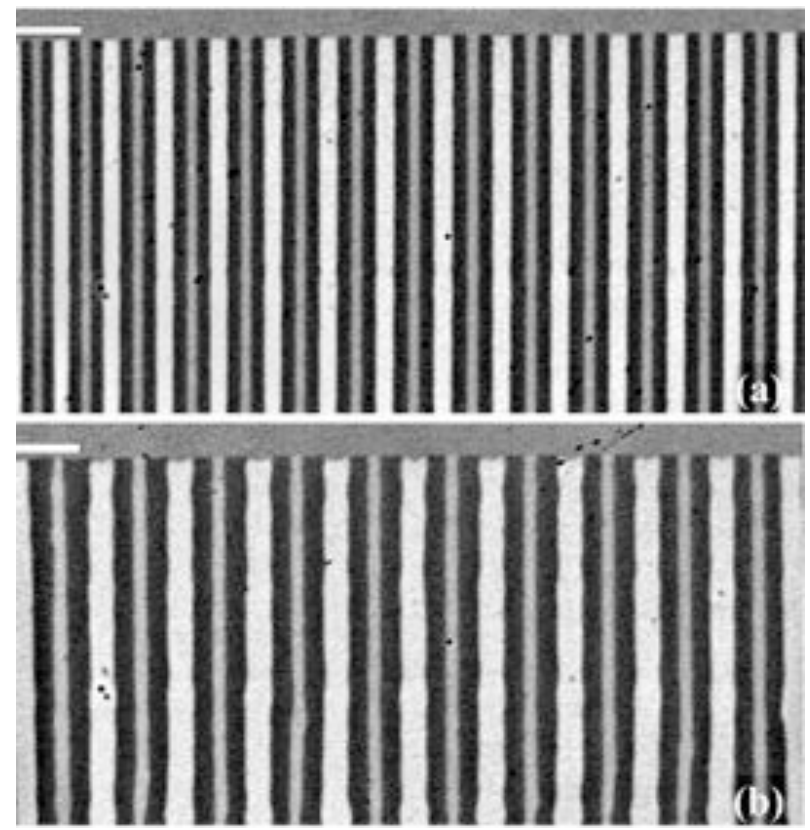

Figure 5: Near-uniform ABAC lamellar patterns observed at $V=0.11 \mu \mathrm{ms}^{-1}$ (bar: $\left.50 \mu \mathrm{m}\right)$, with two different average spacing values: a) $\lambda_{0}=42.5 \mu \mathrm{m}$; b) $\lambda_{0}=60 \mu \mathrm{m}$. 


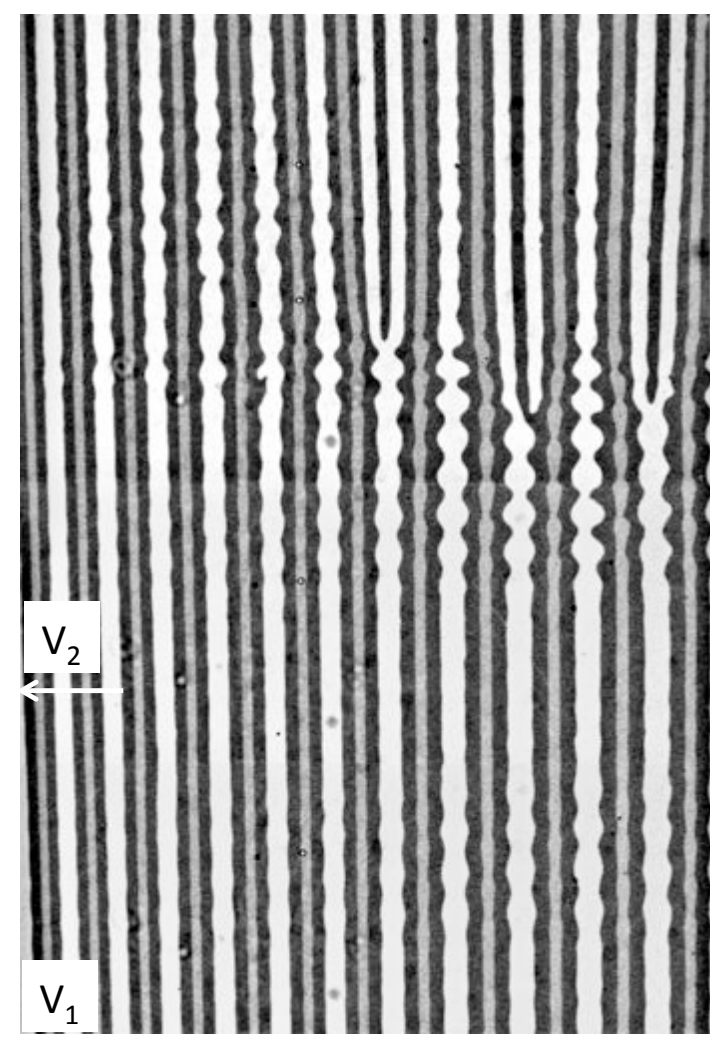

Figure 6: Oscillations and lamella splitting events triggered by two successive velocity-jumps. $V_{0}=0.11 \mu \mathrm{ms}^{-1} . V_{1}=0.13 \mu \mathrm{ms}^{-1} . V_{2}=0.20 \mu \mathrm{ms}^{-1}$. The lowest line of the field of view corresponds to the time of the jump from $V_{0}$ to $V_{1}$. Image width: $610 \mu \mathrm{m}$. 
process after the event (see Section 4.3); (ii) globally, the process resulted in the elimination of a whole repeat unit and thus in a restoration of the ABAC stacking.

\subsection{Spacing diffusion in stable ABAC patterns}

The best method of proving the stability of a dynamical pattern is probably to demonstrate that any imperfection it may contain spontaneously disappears over time. We therefore studied the time evolution of spatial modulations of the spacing profile $\lambda(x)$ in ABAC patterns with a value of the average spacing $\lambda_{0}$ belonging to the stability range, as defined in Fig. 4. In all cases, we indeed observed a uniformisation of $\lambda(x)$. A clear manifestation of this smoothing process could be observed after isolated lamella elimination events, as shown in Fig. 8, and in the upper part of Fig. 7a. This definitely identifies $\lambda_{e l}$ and $\lambda_{b r}$ as being the stability limits of basic ABAC patterns.

A few explanatory remarks will be useful before we present our quantitative results. Let us consider a $\lambda$-distribution consisting of a sinusoidal modulation of amplitude $A$ and wavevector $k$ (or wavelength $L=2 \pi / k$ ) about an average value $\lambda_{0}$. It is a general property of $1 \mathrm{D}$ dynamical patterns that, if $A$ is sufficiently small and $L>>\lambda_{0}$, the time evolution of $\lambda(x)$ is governed by an equation of the form:

$$
\frac{\partial \lambda}{\partial t}=D_{\lambda} \frac{\partial^{2} \lambda}{\partial x^{2}}
$$

where $t$ is time, and $D_{\lambda}$ is a $\lambda_{0}$-dependent coefficient called $\lambda$-diffusion coefficient. The diffusion equation imposes that the amplitude of the $\lambda$-modulation evolves according to

$$
A(t) \propto \exp \left(-D_{\lambda} k^{2} t\right) .
$$

Thus $A(t)$ either increases or decreases exponentially over time depending on the sign of $D_{\lambda}$. It has been shown experimentally and numerically that, in two-phase solidification patterns, $D_{\lambda}$ changes sign at a critical spacing value $\lambda_{c}\left[D_{\lambda}\left(\lambda_{c}\right)=\right.$ $0)$ ], and that the spontaneous amplification of the $\lambda$-modulation that occurs when $\lambda_{0}<\lambda_{c}$ leads to lamella elimination events. This entails that, in thin-DS binary eutectics, $\lambda_{e l}$ and $\lambda_{c}$, while theoretically distinct, cannot be distinguished from one another experimentally. We will show shortly that this is not the case as regards the ABAC patterns.

We performed six independent experimental determinations of $D_{\lambda}$. In that procedure, we chose as an initial time $(t=0)$ a moment at which a peaked modulation of $\lambda$ existed as a result of a recent elimination event, or some other occurrence. The overall results are displayed in Table 1. Two exemplary cases are shown in 

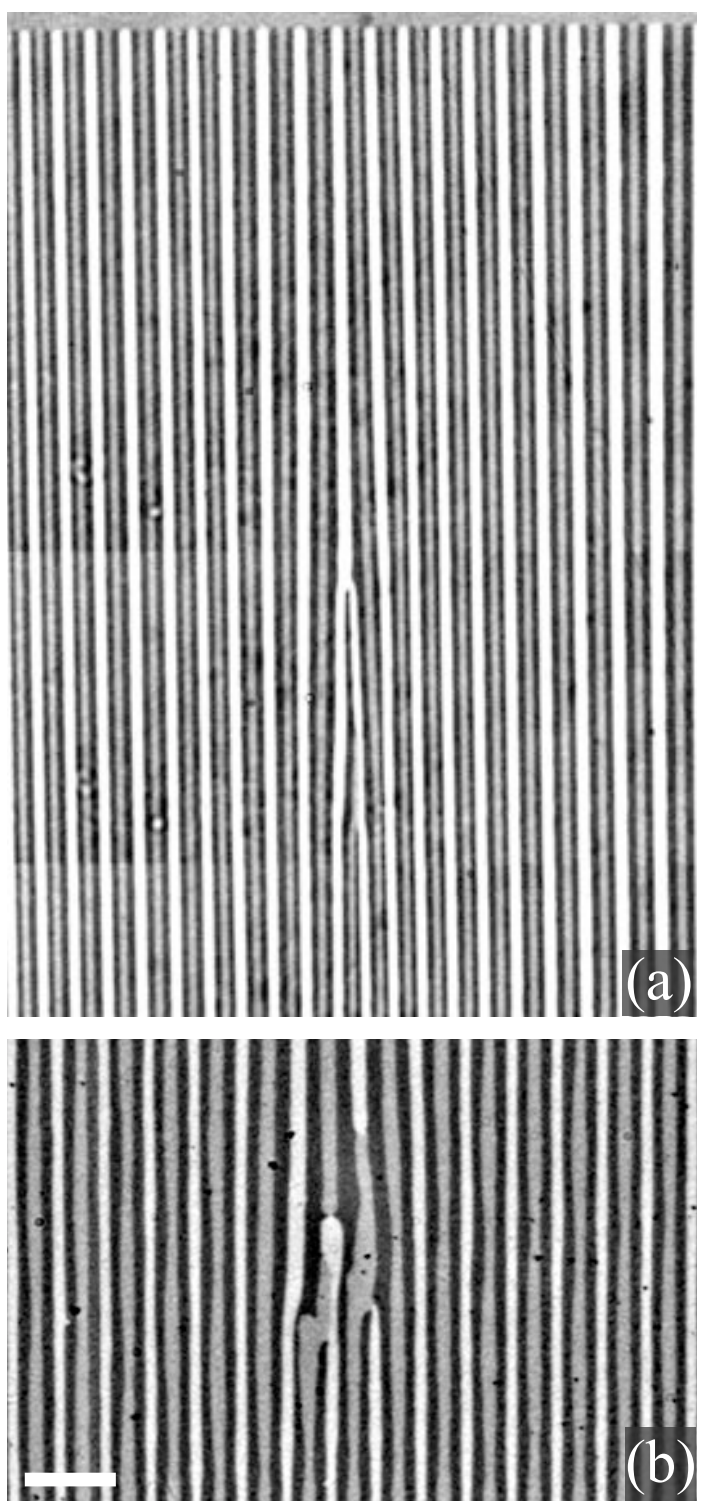

Figure 7: Two different examples of lamella elimination events occurring after a long pulling time at constant velocity. a) $V=0.177 \mu \mathrm{ms}^{-1}$. b) $V=0.21 \mu \mathrm{ms}^{-1}$. Bar: $50 \mu \mathrm{m}$. 


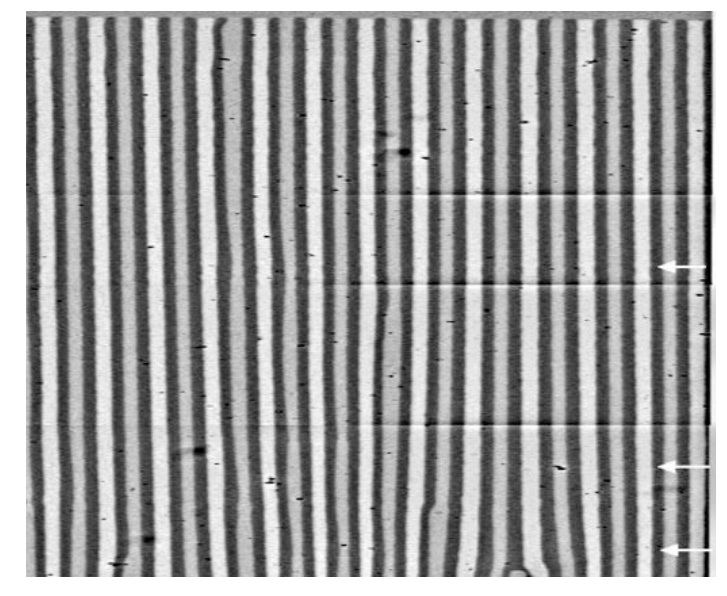

Figure 8: Time evolution of an ABAC pattern in the wake of an elimination event. Arrows: time points corresponding to the lambda plots shown in Fig. 9a. $\mathrm{V}=0.15 \mu \mathrm{ms}^{-1}$. The image has been compressed by a factor of 3 along the growth direction in order to highlight the time evolution of $\lambda(x)$. Image width: $468 \mu \mathrm{m}$

Figure 9. In Fig. 9a (which corresponds to the pattern in Fig. 8), a time series of eight $\lambda(x)$-plots were measured (for clarity, only 3 of them are plotted in Fig. 9a). Functions of the form

$$
\lambda(x)=\lambda_{0}+A_{1}(t) \sin (2 \pi x / L)+A_{2}(t) \sin (2 \pi x /(2 L))
$$

could be fitted satisfactorily to each $\lambda(x)$-plot, thus yielding a set of $A_{1}(t)$ and $A_{2}(t)$ data for a given value of $L(\approx 239 \mu \mathrm{m})$. We then fitted a decreasing exponential function to the $A_{1}(t)$ data, in accordance with Eq. 2 . This yielded $D_{\lambda} \approx 0.85 \pm 0.02$ $\mu m^{2} s^{-1}$. In the second case (Fig. 9b), $\lambda(x)$ measurements were made after the lamella elimination shown in Fig. 7b. Then, the $\lambda(x)$-plots turned out to be best fitted by the gaussian function

$$
\lambda(x)=\lambda_{0}+A(t) \exp \left[-\frac{x^{2}}{\sigma^{2}(t)}\right]
$$

where

$$
A(t)=\frac{\mathrm{A}}{\sqrt{t-t_{0}}}, \quad \sigma(t)=\sqrt{4 D_{\lambda}\left(t-t_{0}\right)} .
$$

which is also a well-known solution of the diffusion equation. In this case, we found $D_{\lambda} \approx 0.43 \pm 0.02 \mu \mathrm{m}^{2} \mathrm{~s}^{-1}$. In overall, the margin of error yielded by the 
best-fit procedures used was about $15 \%$ for all the data. The errors from other origins (grain boundaries, sample edges, curved isotherms) were most probably substantially smaller than this value.

\begin{tabular}{|c|c|c|c|}
\hline$V\left(\mu \mathrm{ms}^{-1}\right)$ & $\lambda_{0}(\mu m)$ & $L(\mu m)$ & $D_{\lambda}\left(\mu m^{2} s^{-1}\right)$ \\
\hline 0.025 & 77.6 & 241 & 0.17 \\
0.035 & 68.4 & 226 & 0.20 \\
$0.046^{*}$ & 49.2 & - & 0.15 \\
0.15 & 36.1 & 239 & 0.85 \\
$0.21^{*}$ & 25.8 & - & 0.43 \\
0.50 & 24.4 & 364 & 7.8 \\
\hline
\end{tabular}

Table 1: Measured values of the $\lambda$-diffusion coefficient $D_{\lambda}$ at the indicated values of $V$ and $\lambda_{0} . L$ : wavelength of the $\lambda$-modulation. ${ }^{*}$ Fit by a gaussian function.

In the case of binary $\mathrm{AB}$ patterns, it has previously been shown that the dependence of $D_{\lambda}$ on $\lambda_{0}$ in the vicinity of $\lambda_{c}$ can be approximated by

$$
D_{\lambda}=\frac{K_{r} \lambda_{0} V^{2}}{G}\left(1-\frac{1}{\Lambda_{0}^{2}}\right)+B V \lambda_{0} \Lambda_{0},
$$

where $\Lambda_{0}=\lambda_{0} / \lambda_{m}, \lambda_{m}=\sqrt{K_{c} / K_{r}} V^{-1 / 2}$, and $B$ is an empirical dimensionless constant [9]. $K_{r}$ and $K_{c}$ are called the Jackson-Hunt constants of the alloy (the coefficient $K_{m}$ introduced in Section 4.1 is equal to $\sqrt{K_{c} / K_{r}}$ ). Let us assume that Eq. 6 is also valid for ABAC patterns, and consider $K_{r}, K_{c}$, and $B$ as adjustable parameters. A least-squares fitting of Eq. 6 to the data of Table 1 yielded the values displayed in Table 2. Due to the dispersion of the experimental data, the error margin is rather large $(\simeq 25 \%)$. In spite of this uncertainty, the data of Table 2 clearly have the same order of magnitude as those generally found in binary eutectics $[9,10]$.

\begin{tabular}{|c|c|c|c|}
\hline $\begin{array}{c}K_{r} \\
{\left[K s \mu m^{-2}\right]}\end{array}$ & $\begin{array}{c}K_{c} \\
{[K \mu m]}\end{array}$ & $B$ & $\begin{array}{c}\lambda_{m}^{2} V \\
{\left[\mu m^{3} s^{-1}\right]}\end{array}$ \\
\hline 0.0166 & 2.23 & 0.054 & 135 \\
\hline
\end{tabular}

Table 2: Best-fit values of the adjustable coefficients of Eq. 6 and of $\lambda_{m}^{2} V=K_{c} / K_{r}$.

According to Eq. 6, there exists, at given $V$ and $G$, a particular value $\lambda_{c}$ of the spacing at which $D_{\lambda}$ vanishes, that is, for $\lambda_{0}<\lambda_{c}$, one has $D_{\lambda}<0$. However, as 

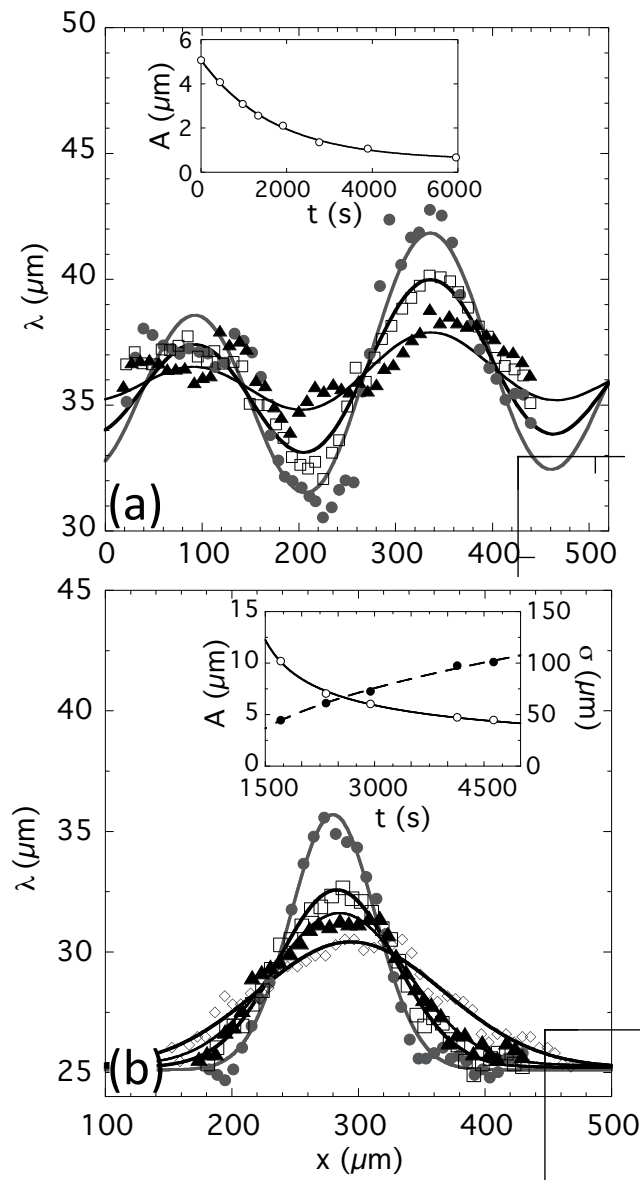

Figure 9: Time series of $\lambda$-plots measured in stable ABAC patterns (the time origin was defined just after a lamella elimination event). (a) $\mathrm{V}=0.15 \mu \mathrm{ms}^{-1}$ (same experiment as in Fig. 8). Circles: $\mathrm{t}=440$ s. Squares: $\mathrm{t}=1340 \mathrm{~s}$. Triangles: $\mathrm{t}=3890 \mathrm{~s}$. Thick lines: best-fitting curves to the $\lambda$-plots using Eq. 3. Inset: Decreasing exponential function fitted to $A_{1}(t)$ using Eq. 2. (b) $\mathrm{V}=$ $0.21 \mu \mathrm{ms}^{-1}$ (same experiment as in Fig. $7 \mathrm{~b}$ ). Circles: $\mathrm{t}=1710 \mathrm{~s}$. Squares: $\mathrm{t}=2330 \mathrm{~s}$. Triangles: $\mathrm{t}$ $=2940$ s. Diamonds: $\mathrm{t}=4130 \mathrm{~s}$. Thick lines: best-fitting curves to the $\lambda$-plots using Eq. 4. Inset: best-fitting curves to $A(t)$ (continuous line) and $\sigma(t)$ (broken line) using Eq. 5. 
mentioned above, we did not observe the amplification of a $\lambda$ modulation, even in those ABAC patterns, in which lamella elimination occurred. This contrasts to what has been observed previously in two-phase patterns during thin-DS of various binary-eutectic alloys. Therefore, we are led to conclude that, in ABAC growth patterns, the threshold $\lambda_{e l}$ for lamella elimination is not related to $\lambda_{c}$. We obtained a $\lambda_{c}(V)$ curve by plugging the data of Table 2 into Eq. 6, and setting $D_{\lambda}=0$. This curve has been plotted in Fig. $4 \mathrm{~b}$ as a dashed line. As can be seen, the calculated values of $\lambda_{c}$ are close to, and probably smaller than $\lambda_{e l}$. This supports the view that a $\lambda$-diffusion instability exists, but is hidden by the instability process leading to lamella elimination. What then was this process? Close-up examinations of the lamella elimination events were only capable of showing that it was very fast and highly localized. To sum up, lamella elimination was triggered by a short-wavelength instability occurring at a $\lambda$-value slightly larger than $\lambda_{c}$. The same has already been observed in other 1D dynamical systems (for instance cellular solidification patterns, see [18]). In our case, the instability involved seems to have a wavelength shorter than $\lambda$ (i.e. the width of the ABAC motif).

\subsection{ABC patterns and complex patterns}

In addition to the ABAC patterns, we also observed, under certain conditions, other types of extended growth patterns. Recent numerical simulations demonstrated the possible existence of stationary patterns with an $\mathrm{ABC}$ repeat unit in ternary eutectics [4]. We indeed observed small domains of $\mathrm{ABC}$ patterns in the In- $\mathrm{In}_{2} \mathrm{Bi}$-Sn eutectic, but only as transients following large-amplitude $V$-jumps (Fig. 10). Interestingly, these patterns were drifting laterally, in accordance with the fact that the ABC motif does not have a mirror symmetry. The order of magnitude of the drift angles observed was $10^{\circ}$ with respect to the growth direction.

We also observed three-phase growth patterns with a large variety of superstructures, which remained stable over the duration of the experiments. The simplest of these superstructures was $[\mathrm{AB}]_{2} \mathrm{AC}$ (see Fig. 6). Patterns with much wider superstructures could be obtained by applying a particular velocity program during the melting-annealing-invasion procedure (see Section 3). Two examples, one with an $[\mathrm{AB}]_{2}[\mathrm{AC}]_{2}$ motif, the other with an $[\mathrm{AB}]_{4}[\mathrm{AC}]_{6} \mathrm{~B}$ motif are shown in Fig. 11. These patterns, although essentially periodic, contained numerous (stationary) "stacking faults". We note the presence of stationary BC interfaces in Fig. $11 \mathrm{~b}$, which was exceptional. The stability of such patterns is somewhat surprising given that an $[\mathrm{AB}]_{\mathrm{m}}[\mathrm{AC}]_{\mathrm{n}}$ pattern actually consists of wide adjacent two-phase domains, which, under other conditions, could invade each other. The explanation 

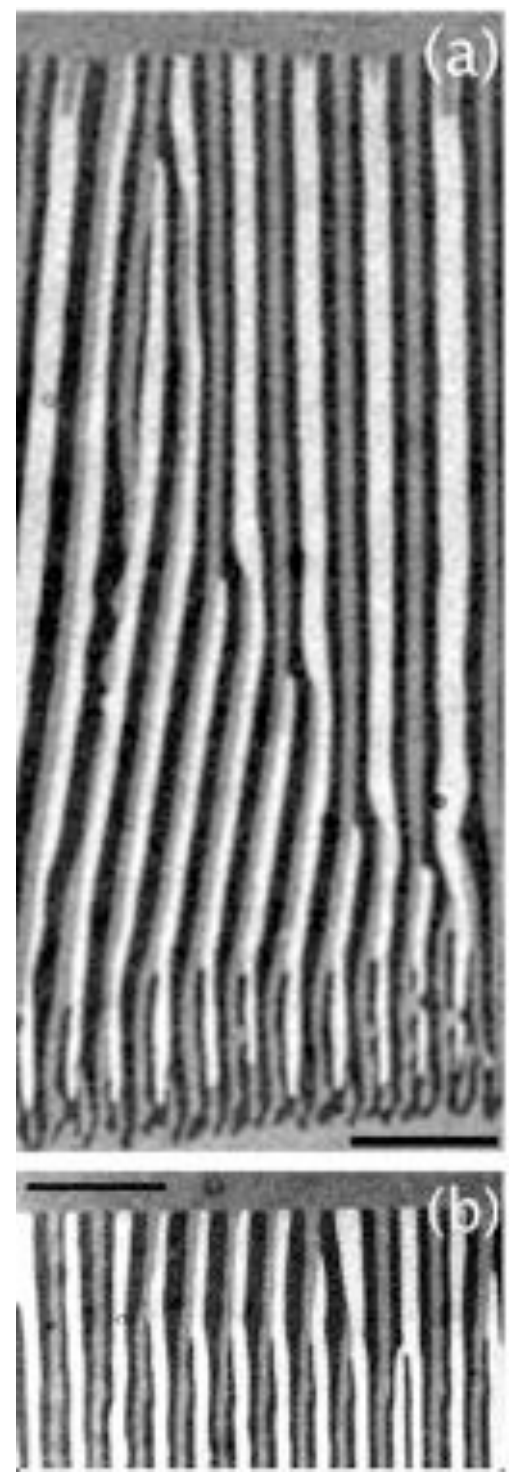

Figure 10: Transient $\mathrm{ABC}$ patterns observed in two distinct experiments: (a) a short time after the onset of pulling $\left(V=0.531 \mu \mathrm{ms}^{-1}\right)$; (b) after a velocity decrease of a factor $10\left(V=0.071 \mu \mathrm{ms}^{-1}\right)$. Bars: $50 \mu \mathrm{m}$. 
probably lies in the detailed local structure of the boundary between the two-phase domains, which remains to be studied.
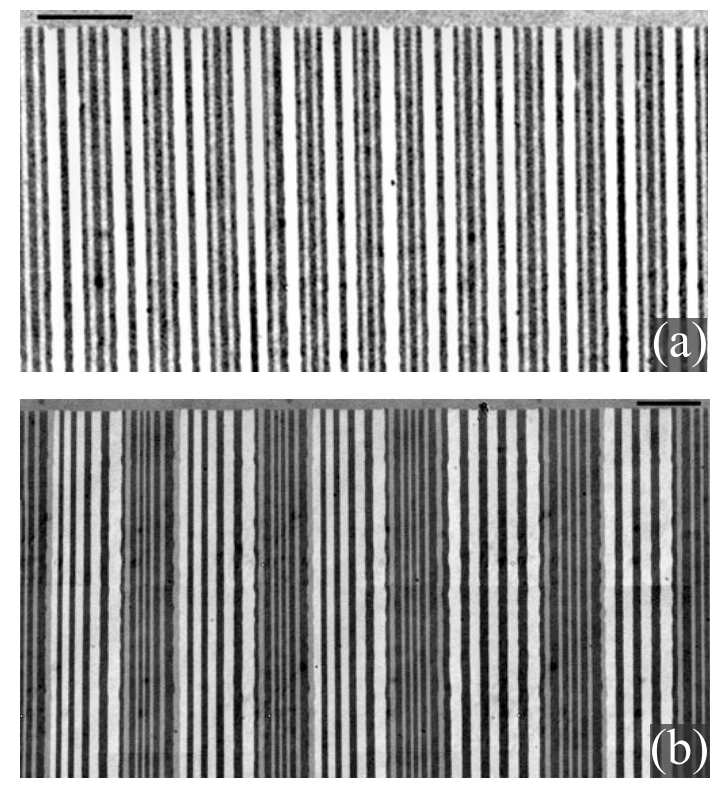

Figure 11: Stationary growth patterns with complex superstructures. (a) $V=0.247 \mu \mathrm{ms}^{-1}$. (b) $V=0.177 \mu \mathrm{ms}^{-1}$. Bars: $100 \mu \mathrm{m}$.

\section{Conclusion}

We have shown experimentally that three-phase ABAC growth patterns in thin-sample directional solidification of a ternary eutectic alloy have a finitewidth stability range of spacing. The upper stability limit corresponds to lamellar branching, and the lower limit to lamella elimination. We have measured the value of the $\lambda$-diffusion coefficient in a series of stable patterns with different $V$ and $\lambda$ values in the particular case of the $\mathrm{In}_{-} \mathrm{In}_{2} \mathrm{Bi}-\mathrm{Sn}$ eutectic. We have evaluated the ABAC Jackson-Hunt constant of the alloy to be $\lambda_{m}^{2} V=135 \pm 20 \mu \mathrm{m}^{3} \mathrm{~s}^{-1}$. In terms of the reduced spacing $\Lambda=\lambda / \lambda_{m}$, the upper instability threshold was shown to be independent of $V\left(\Lambda_{\text {sup }}=1.9 \pm 0.2\right)$. The lower instability threshold $\Lambda_{\text {inf }}$ (of about 0.6 at the smallest pulling velocity used in this study, that is, $V=0.01$ $\mu m s^{-1}$ ) increases with $V$, and is roughly equal to 1 above $V=0.2 \mu \mathrm{ms}^{-1}$. In Ref [2], Witusiewicz et al. have measured an average value $\langle\lambda\rangle$ of the $\mathrm{ABAC}$ 
spacing in the $\mathrm{In}-\mathrm{In}_{2} \mathrm{Bi}-\mathrm{Sn}$ eutectic under the same thermal gradient as we used, over a large $V$ range. Using the above mentioned estimate of $\lambda_{m}$, we may sum up their results as $\langle\Lambda\rangle \approx 1.5$ at high $V$, which is well inside the stability range we have measured and hence agrees with the results of this study. This investigation opens up new questions such as: What is the precise nature of the instability process which triggers lamella elimination and thereby determines the value of $\lambda_{\text {inf }}$ in ABAC patterns? How can three-phase patterns with a complex superstructure grow in a stationary way? Numerical studies will probably be necessary in order to elucidate these questions.

\section{Acknowledgements}

We thank Patricia Ott for her help in the preparation of the samples. We thank the French Center for Spatial Research (CNES) for financial support. This work was financially funded by TÜBITAK 3501 (Grant no: 212M013) and European Commission Marie Curie Career Integration Grant FP7-PEOPLE-2012-CIG (NEUSOL 334216) for authors MS and SY. One of us (SY) benefited from an Erasmus grant.

[1] K. A. Jackson, J. D. Hunt, Lamellar and rod eutectic growth, Transactions of the Metallurgical Society of AIME 236 (1966) 1129.

[2] V. T. Witusiewicz, U. Hecht, S. Rex, M. Apel, In situ observation of microstructure evolution in low-melting bi-in-sn alloys by light microscopy, Acta Materialia 53 (13) (2005) 3663-3669.

[3] V. T. Witusiewicz, L. Sturz, U. Hecht, S. Rex, Phase equilibria and eutectic growth in quaternary organic alloys amino-methyl-propanediol(d)camphor-neopentylglycol-succinonitrile (ampd-dc-npg-scn), Journal of Crystal Growth 297 (1) (2006) 117-132.

[4] A. Choudhury, M. Plapp, B. Nestler, Theoretical and numerical study of lamellar eutectic three-phase growth in ternary alloys, Physical Review E $83(5)$.

[5] W. Boettinger, Surface relief cinemicrography of the unsteady solidification of the lead-tin-cadmium ternary eutectic, Ph.D. thesis, Jonhs Hopkins University (1972). 
[6] M. A. Ruggiero, J. W. Rutter, Origin of microstructure in the 332 k eutectic of the bi-in-sn system, Materials Science and Technology 13 (1) (1997) 511.

[7] A. Karma, A. Sarkissian, Morphological instabilities of lamellar eutectics, Metallurgical and Materials Transactions a-Physical Metallurgy and Materials Science 27 (3) (1996) 635-656.

[8] M. Ginibre, S. Akamatsu, G. Faivre, Experimental determination of the stability diagram of a lamellar eutectic growth front, The American Physical Society- Physical Review E 56 (1) (1997) 780.

[9] S. Akamatsu, M. Plapp, G. Faivre, A. Karma, Overstability of lamellar eutectic growth below the minimum-undercooling spacing, Met. Mat. Trans 35A (2004) 1815-1828.

[10] S. Akamatsu, S. Bottin-Rousseau, G. Faivre, Determination of the jacksonhunt constants of the in-in(2)bi eutectic alloy based on in situ observation of its solidification dynamics, Acta Materialia 59 (20) (2011) 7586-7591.

[11] P. Manneville, Dissipative structures and weak turbulence, Academic Press.

[12] V. T. Witusiewicz, U. Hecht, B. Bottger, S. Rex, Thermodynamic reoptimisation of the bi-in-sn system based on new experimental data, Journal of Alloys and Compounds 428 (1-2) (2007) 115-124.

[13] S. Akamatsu, S. Bottin-Rousseau, M. Perrut, G. Faivre, V. T. Witusiewicz, L. Sturz, Real-time study of thin and bulk eutectic growth in succinonitrile(d)camphor alloys, Journal of Crystal Growth 299 (2) (2007) 418.

[14] B. Caroli, C. Caroli, G. Faivre, J. Mergy, Lamellar eutectic growth of cbr4c2c16: effect of crystal anisotropy on lamellar orientations and wavelength dispersion, Journal of Crystal Growth 118 (1992) 135-150.

[15] S. Akamatsu, S. Bottin-Rousseau, M. Şerefoğlu, G. Faivre, A theory of thin lamellar eutectic growth with anisotropic interphase boundaries, Acta Materialia 60 (6-7) (2012) 3199-3205.

[16] S. Akamatsu, S. Moulinet, G. Faivre, The formation of lamellar-eutectic grains in thin samples, Metallurgical And Materials Transactions A 32A (2001) 2039-2048. 
[17] S. Rex, B. Bottger, V. Witusiewicz, U. Hecht, Transient eutectic solidification in in-bi-sn: Two-dimensional experiments and numerical simulation, Materials Science And Engineering A-Structural Materials Properties Microstructure And Processing 413 (2005) 249-254.

[18] P. Kopczynski, W. J. Rappel, A. Karma, Critical role of crystalline anisotropy in the stability of cellular array structures in directional solidification, Physical Review Letters 77 (16) (1996) 3387-3390. 